\title{
Unusual Case of Pneumothorax Caused by Costal Osteochondroma
}

\author{
Rahul Vemula ${ }^{1}$, Sarav Shah ${ }^{2}$, Lournes J. Willekes $\mathbf{I I}^{3^{*}}$ \\ ${ }^{1}$ Department of Surgery, Monmouth Medical Center, Long Branch, USA; ${ }^{2}$ Drexel University College of Medicine, Philadelphia, \\ USA; ${ }^{3}$ Division of Thoracic Surgery, Monmouth Medical Center, Long Branch, USA. \\ Email: *Lwj898@hotmail.com
}

Received July $31^{\text {st }}$, 2012; revised September $1^{\text {st }}$, 2012; accepted September $10^{\text {th }}, 2012$

\begin{abstract}
Osteochondroma is the most common benign cartilage tumor. Although, most lesions are asymptomatic, life-threatening conditions caused osteochondromas have been reported in the literature such pneumothorax, hemothorax, diaphragmatic rupture, and lung injury. We report a case of a thirty year old male who presented with shortness of breath and was found to have a pneumothorax on chest X-ray. Subsequent Computed Tomography of the chest revealed a 1.3 $\mathrm{cm}$ growth on the posterior portion of the fourth right rib protruding into the right middle lobe. The patient underwent thoracoscopic resection of the lesion along with a wedge resection of the right middle lobe. The rib was reconstructed using a metal plate. He remains asymptomatic without recurrence on a one year follow up.
\end{abstract}

Keywords: Osteochondroma; VATS; Thoracoscopy; Thoracic Oncology

\section{Introduction}

Osteochondroma is the most common benign cartilage tumor [1]. Most often the lesions are incidental, asymptomatic findings on routine radiographs or on radiographs to assess other conditions [2]. Although most patients are asymptomatic, there have been life-threatening conditions such as diaphragmatic, pericardial, and lung injury reported [3-9]. We report a case of a thirty year old man presenting with a spontaneous pneumothorax secondary to a costal osteochondroma that eroded through the middle lobe of the lung.

\section{Case Report}

A tall thirty year old male with an athletic build presents to his primary care physician with acute onset of right sided chest pain associated with moderate shortness of breath. The patient originally thought he was having difficulty with his seasonal allergies but the symptoms did not resolve with medical treatment that included over the counter antihistamines. After chest $\mathrm{x}$-ray revealed a right sided pneumothorax, he was sent to the emergency department. In the emergency department he was short of breath and on physical exam he had diminished breath sounds on the right with no wheezes, rhonchi, or rales on the unaffected side. Medical, social, and family histories were unremarkable.

\footnotetext{
*Corresponding author.
}

A computed tomography of the chest without contrast revealed a small right pneumothorax and a 1.3 centimeter growth along the anterior aspect of the right fourth rib (Figure 1). The growth projected into the middle lobe and had created a pulmonary scar immediately adjacent. Because of symptoms, pneumothorax, and potential for bleeding, he was scheduled to have the mass removed.

The patient was taken to operating room for right partial rib resection with reconstruction, wedge resection, and mechanical pleurodesis. He was placed under general anesthesia with a double lumen tube and positioned in a forty-five degree upward position with the right chest upward. Using video thoracoscopy, two ten-millimeter

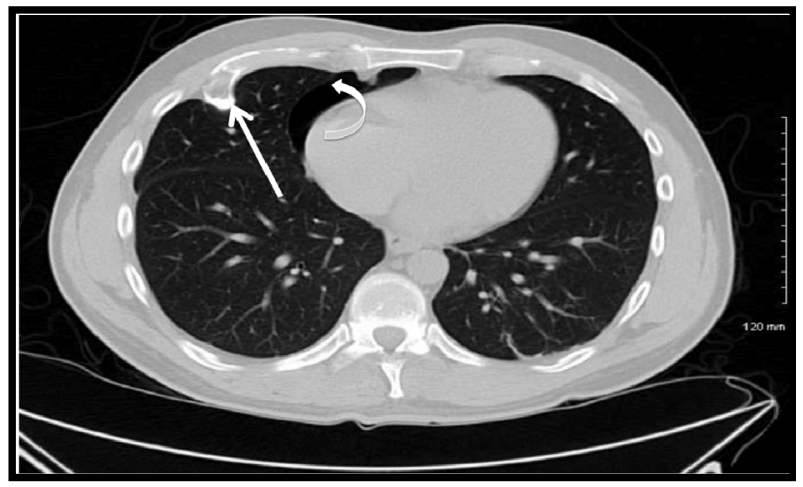

Figure 1. Computed tomography of chest without contrast showing lesion on posterior aspect of 4 th rib (white arrow), and pneumothorax (curved white arrow). 


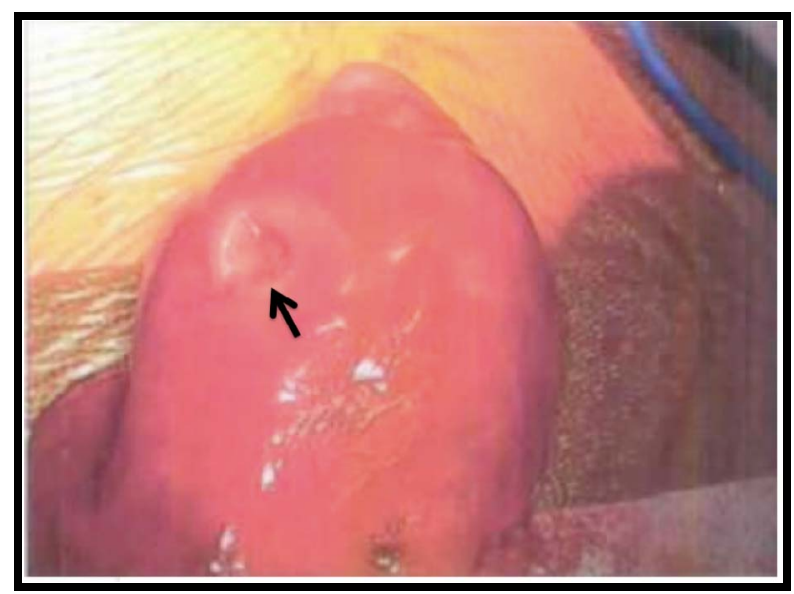

Figure 2. Portion of right middle lobe showing area of erosion from tumor (black arrow).

working ports were made and a ten millimeter thirty degree viewing scope was used. The patient had adhesions from the chest wall growth to the middle lobe. The bony tumor was noted to have eroded through the anterior to the posterior aspect of the middle lobe (Figures $\mathbf{2}$ and 3). It was marked from the outside of the chest wall. A counter incision directly over the rib was made. This was carried down to the periosteum. A subperiosteal resection of the rib with rib division laterally and medially was completed (Figure 4). A stainless steel bar (curved both longitudinally and rotationally) was fashioned to reconstruct the rib. This was anchored into place medially and laterally with locking screws (Figure 5). Wedge resection of the damaged portion of the middle lobe was completed. Thoracoscopic mechanical pleurodesis was accomplished by abrasing the parietal pleura with an electrocautery scratch pad until petechiae was observed. A \#32 French pleural drain was placed.

Pathologic examination of the resected specimen confirmed osteochondroma (Figure 6). Lung pathology showed chronic inflammation with absence of the visceral pleura over $20 \%$ of the specimen.

Postoperatively, the patient's recovery was uneventful. A chest $\mathrm{x}$-ray 2 months later revealed an intact bar anteriorly, no pneumothorax, and clear lung fields. The patient has been symptom free and has had no recurrence on a one year follow up.

\section{Discussion}

Approximately half of all primary chest wall tumors are benign. Most of the benign neoplasms are of cartilaginous origin, with osteochondroma (exostosis) being the most common [2]. There are two types of costal exostosis: hereditary multiple exostoses and solitary costal exostosis [3]. Most often they are asymptomatic, but rarely are they associated with pneumothorax, hemotho-

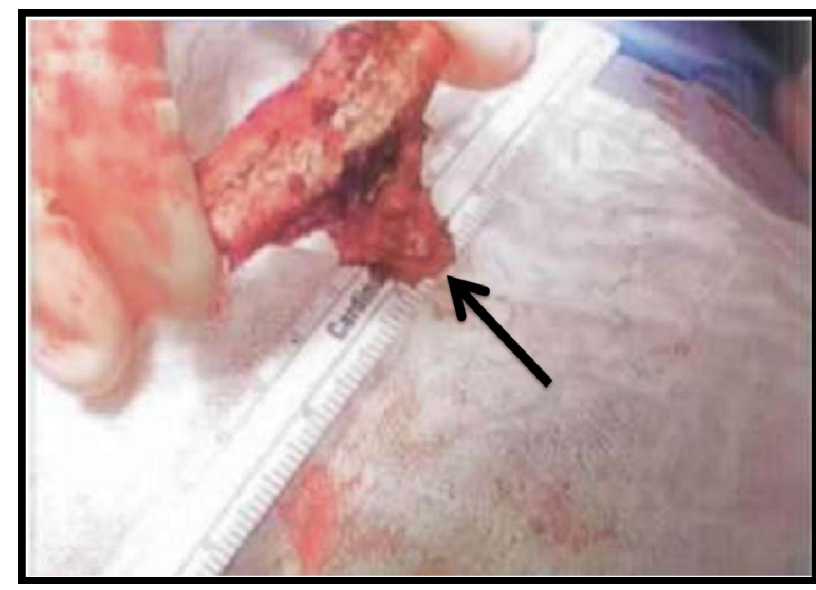

Figure 3. Resected portion of 4th right rib containing tumor on the posterior surface (black arrow).

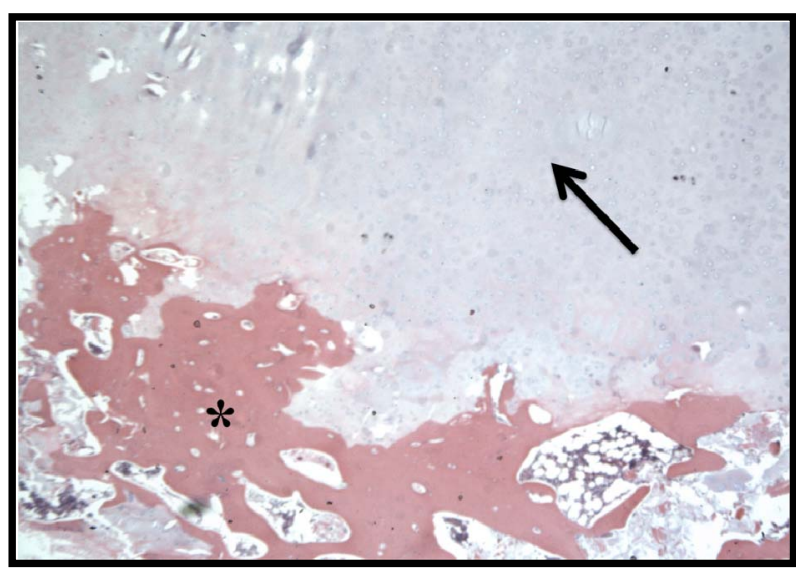

Figure 4. Pathologic examination of the specimen showing normal bone with overlying cartilage (black arrow) showing disorganized growth pattern (asterisk) characteristic of osteochondroma.

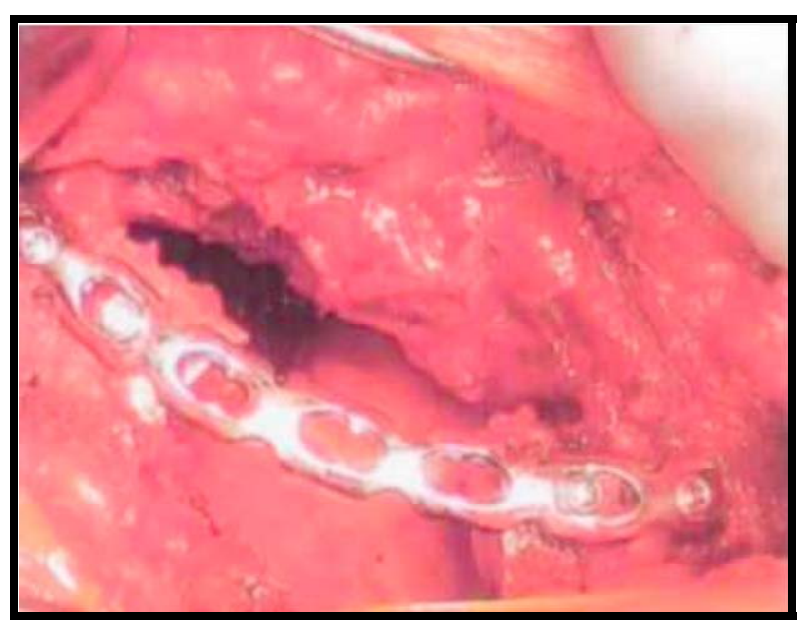

Figure 5. Right 4th rib reconstructed with metal plate.

rax, diaphragmatic rupture and empyema [3-5]. Many of these neoplasms have radiographic characteristics on 


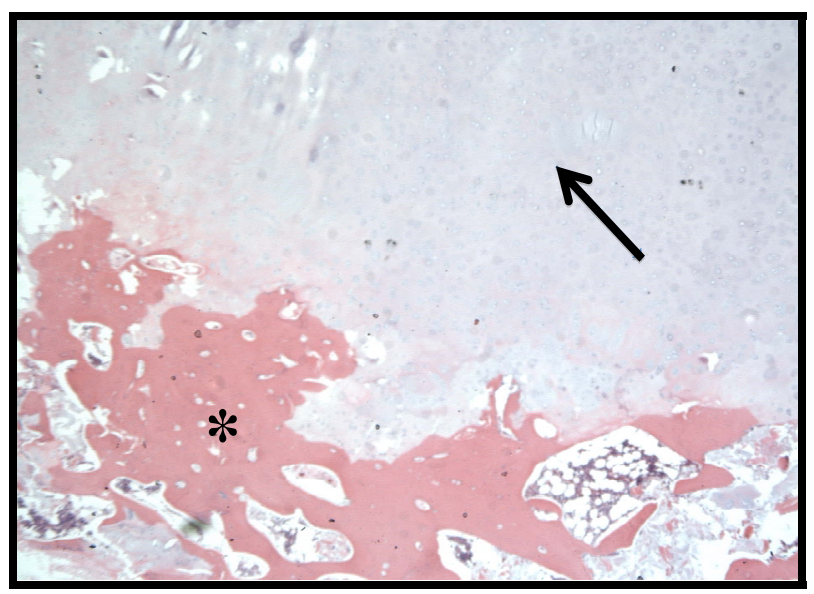

Figure 6. Pathologic examination of the specimen showing normal bone with overlying cartilage showing disorganized growth pattern characteristic of osteochondroma.

plain films, although other imaging such as CT or MRI may be needed to determine the most likely diagnosis. Once the tumor is detected the physician may elect to observe it. If this course is taken, there must be careful surveillance because, although rare, malignant transformation does occur [2]. One to two percent of solitary osteochondromas become malignant [1]. That number goes to five to twenty percent if associated with multiple hereditary exostoses. Changes in the lesion accompanied by discomfort should arouse the physician's suspicion of malignant degeneration [2].

Spontaneous pneumothorax is most commonly caused by the unprompted rupture of a pulmonary bleb. In our case there was little evidence of bleb disease, but rather a causative factor from the protruding chest wall tumor into the lung parenchyma. Thoracoscopic resection of such lesions has been described in the literature $[10,11]$, and VATS guided resection proved very beneficial and diminished his post-operative pain and allowed for excellent vision of all pathology. Given the anterior location of the osteochondroma and the patient's athletic build, rib reconstruction was performed to stabilize the chest wall.

\section{REFERENCES}

[1] V. Devita, S. Hellman, S. Rosenberg, et al., "Cancer Principles and Practice of Oncology,” 7th Edition, Lippincott
Williams and Wilkens, New York, 2005, p. 1638.

[2] F. G. Pearson, J. Cooper, J. Deslauriers, et al., "Thoracic Surgery," 2nd Edition, Churchill Livingstone, New York, 2002, p. 1340.

[3] A. Khosla and R. Parry, "Costal Osteochondroma Causing Pneumothorax in an Adolescent: A Case Report and Review of the Literature," Journal of Pediatric Surgery, Vol. 45, No. 11, 2010, pp. 2250-2253. doi:10.1016/j.jpedsurg.2010.06.045

[4] W. Hajjar, Y. El-Medany, M. A. Essa, M. A. Rafay, M. H. Ashour and K. M. Al-Kattan, "Unusual Presentation of rib Exostosis,” The Annals of Thoracic Surgery, Vol. 75, No. 2, 2003, pp. 575-577. doi:10.1016/S0003-4975(02)04326-6

[5] D. A. Simansky, M. Paley, A. Werczberger, Y. B. Ziv and A. Yellin, "Exostosis of a Rib Causing Laceration of the Diaphragm: Diagnosis and Management," The Annals of Thoracic Surgery, Vol. 63, No. 3, 1997, pp. 856-857. doi:10.1016/S0003-4975(96)01269-6

[6] W. Jin, S. Y. Syun, E. Ryoo, Y. S. Lim and J. K. Kim, "Costal Osteochondroma Presenting as Haemothorax and Diaphragmatic Laceration,” Pediatric Radiology, Vol. 35, No. 7, 2005, pp. 706-709. doi:10.1007/s00247-005-1407-9

[7] K. G. Buchan, V. Zamvar, K. M. Mandana, E. Nihal and P. Kulatilake, "Juxtacardiac Osterochondroma Presenting as Recurrent Haemothorax," European Journal of CardioThoracic Surgery, Vol. 20, No. 1, 2001, pp. 208-210. doi:10.1016/S1010-7940(01)00721-7

[8] G. Gulino, A. Di Martino, N. Papapietro, E. Taglieri and V. Denaro, "Rib Osteochondroma in the Adolescent: Case Report and Survey of Complications," Journal of Orthopaedics and Traumatology, Vol. 5, No. 2, 2004, pp. 128131.

[9] A. Asmat and J. Tam, "Spontaneous Haemothorax from an Osteochondroma,” European Journal Cardio-Thoracic Surgery, Vol. 36, No. 2, 2009, pp. 394-396. doi:10.1016/j.ejcts.2009.04.039

[10] M. Alifano, M. Morcos, M. Thierry and J. Regnard, “An Unusual Case of Hiccup: Costal Exostosis. Treatment by Video-Assisted Thoracic Surgery,” European Journal Cardio-Thoracic Surgery, Vol. 23, No. 6, 2003, pp. 10561058. doi:10.1016/S1010-7940(03)00082-4

[11] Y. Matsuno, Y. Mori, Y. Umeda and I. Takiya, “Thoracoscopic Resection for Costal Exostosis Presenting with Hemothorax in a Child," European Journal of Pediatric Surgery, Vol. 19, No. 4, 2009, pp. 251-271. doi:10.1055/s-2008-1038884 